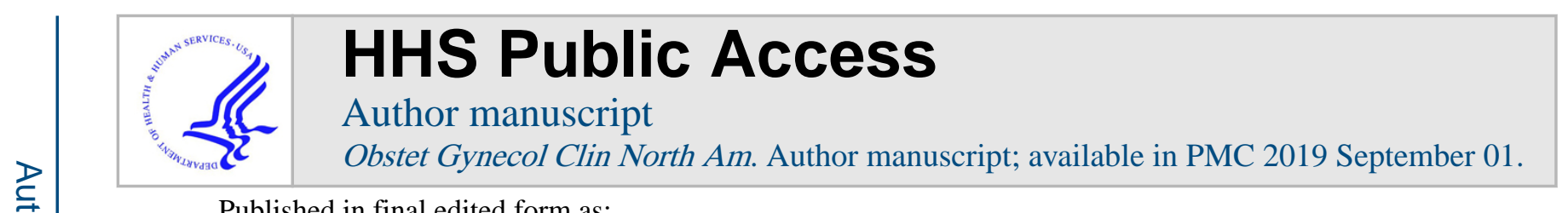

Published in final edited form as:

Obstet Gynecol Clin North Am. 2018 September ; 45(3): 403-417. doi:10.1016/j.ogc.2018.05.002.

\title{
Treatment of Peripartum Bipolar Disorder
}

\author{
Crystal T. Clark, MD, MSc ${ }^{*}$ and Katherine L. Wisner, MD, MS \\ Department of Psychiatry and Behavioral Sciences, Northwestern University Feinberg School of \\ Medicine, 676 St. Clair Street, Chicago, IL 60611, USA
}

\author{
Keywords \\ Bipolar disorder; Pregnancy; Lithium; Lamotrigine; Carbamazepine; Antipsychotics; Light \\ therapy; MDQ
}

\section{INTRODUCTION}

Bipolar disorder (BD) is characterized by chronic remitting and relapsing episodes of depression, hypomania, and mania. The lifetime prevalence is $4.4 \%$ (including all sub-types) of the United States population. ${ }^{1}$ Men and women have a similar incidence of BD, but women are more likely to have depressive episodes, precipitous changes between depression and hypomania/mania (ie, rapid cycling), and episodes of both depressive and manic symptoms (ie, mixed states). ${ }^{2}$ With an average age of onset at 18 years, women are affected throughout their reproductive years and pregnancy is a vulnerable time for episode recurrence. The mainstay of treatment for $\mathrm{BD}$ is pharmacotherapy and the goal is prevention of symptoms of BD during pregnancy and postpartum.

Compared with women with major depressive disorder, those with BD are at a greater risk for mood worsening immediately postpartum and are $50 \%$ more likely than those with major depression to have postpartum depression. ${ }^{3}$ Women with BD are seven times more likely to be hospitalized for a first-time mood episode early postpartum. ${ }^{4}$ Mental illness in the perinatal period increases the risk for suicide, a leading cause of maternal death. ${ }^{5,6}$ With a $25 \%$ to $50 \%$ increased risk for psychosis - a 100-fold increase over the rate in the general population-women with BD are particularly vulnerable to severe postpartum mood worsening. ${ }^{3}$ Clinicians must be able to distinguish between unipolar and bipolar depressive episodes to provide appropriate clinical management.

Optimizing pregnancy and postpartum outcomes for women with BD requires early identification, symptom monitoring, and effective treatment. This review focuses on the risk of untreated perinatal BD and treatment options. We discuss:

1. Screening for perinatal BD,

2. The risks of illness exposure,

*Corresponding author. crystal.clark@ northwestern.edu.

Disclosure Statement: The authors have no conflicts to disclose. 
3. The risks of pharmacotherapy during pregnancy and breastfeeding,

4. Effective dosing across childbearing, and

5. Nondrug treatments including bright light therapy and electroconvulsive therapy.

\section{PATIENT EVALUATION OVERVIEW}

Although manic and hypomanic episodes are diagnostic of BD, most episodes in the perinatal period are depressive. Acute episodes of bipolar and unipolar depression are clinically indistinguishable. The differentiation is based on the occurrence of previous manic or hypomanic episodes, which define bipolar 1 and bipolar 2 disorder, respectively. The distinction is important in the postpartum period, which confers a high risk for both recurrent and new onset BD. Manic and hypomanic episodes are distinguished by an elevated or irritable mood and increased energy that is present most of every day for four (hypomania) or seven (mania) consecutive days. ${ }^{6}$ Patients must also have at least three or four (if an irritable mood is present) additional symptoms present including grandiosity, sleeping less than usual or not at all, rapid and verbose speech, racing thoughts, difficulty focusing, impulsive behavior, and/or increased goal directed activity at home or at work. ${ }^{6}$ The onset of mood symptoms and the change in energy must not be attributable to the use of a substance or a medical comorbidity to meet criterion for BD. A personal history of postpartum depression, postpartum psychosis, or a family history of a first-degree relative with $\mathrm{BD}$ increases the risk for illness onset during the perinatal period. Ideally, during a preconception appointment, clinicians will inquire about diagnoses of mental illness, treatment with psychotropic medications, and/or family psychiatric history to guide the discussion of pregnancy management.

The US Preventive Services Task Force recommends depression screening for pregnant and postpartum women, but it does not mention strategies to differentiate unipolar from bipolar depression. ${ }^{7}$ In a study by Wisner and colleagues ${ }^{8}$ of 10,000 postpartum women in an urban obstetric setting, $22.6 \%$ of women with a positive depression screen (score of $\geq 10$ on the Edinburgh Postnatal Depression Scale [EPDS]) were diagnosed with BD with a research diagnostic examination. None of the commonly used depression screens, such as the EPDS ${ }^{9}$ (a 10-item, self-report scale, translated into 36 languages) and Patient Health Questionnaire (PHQ-9 ${ }^{10}$; a9-item, self-report instrument that has been validated in perinatal clinics) distinguish bipolar from unipolar depression.

The Mood Disorder Questionnaire (MDQ) ${ }^{11}$ is a brief self-report screen for BD that takes approximately 5 minutes to complete. The MDQ includes 13 symptoms, the timing of symptoms, and the degree of impairment. A positive screen includes endorsement of seven symptoms occurring at the same time with moderate or serious impairment. Similar to the findings of Wisner and colleagues, ${ }^{8}$ Merrill and colleagues ${ }^{12}$ reported that $21.4 \%$ of perinatal women in an obstetric clinic screened with the EPDS screened positive for BD on the MDQ. In a study of postpartum women with a positive EPDS score (a score of $\geq 10$ ), Clark and colleagues ${ }^{13}$ foundthat the MDQ accurately identified $50 \%$ of women with BD according to a structured diagnostic interview. The identification rate was increased to $68 \%$ by excluding the impairment criterion. We recommend using the following MDQ screen 
criteria for perinatal women: (1) the presence of 7 symptoms and (2) endorsement that symptoms occurred at the same time. We advise screening with both the EPDS or PHQ-9 and the MDQ before treatment is initiated. Screening patients with the MDQ at the initial presentation during pregnancy and again at the first follow-up visit with an obstetrician increases detection of patients with BD. Although the MDQ includes questions about a history of manic symptoms, it is also a useful way to determine the presence of current symptoms.

Psychiatric evaluation is the next step for patients who screen positive on the MDQ. When psychiatric services are unavailable or will be delayed, obstetricians are in the best position to initiate treatment. A review of current or past effective medication regimens informs the treatment plan. Patients who are effectively treated may elect to continue their medication regimen to prevent episode recurrence. Continuation of pharmacotherapy will be most critical for patients who endorse rapid decompensation when medications are discontinued, hospitalizations for BD episodes, suicide attempts, psychotic episodes, poor response to medication trials, and/or long recovery times when resuming effective treatment regimens. For positive screens without a prior diagnosis, the MDQ is a useful tool to educate patients about BD symptoms.

After birth, women who have a diagnosis of BD or screen positive on the MDQ and who cannot access psychiatric care can be supported through follow-up with their obstetrician for symptom monitoring with the EPDS/PHQ-9 and MDQ as well as medication management. The function of monitoring is to assess for the onset or worsening of symptoms given the risk of postpartum psychosis, a psychiatric emergency, among women with BD (refer to Lauren M. Osborne's article, "Recognizing and Managing Postpartum Psychosis: A Clinical Guide for Obstetric Providers," in this issue)

\section{PHARMACOLOGIC TREATMENT OPTIONS DURING PREGNANCY AND LACTATION}

The choice to continue medication during pregnancy balances the risks of an untreated illness with the risks of medication exposure (Fig. 1). Untreated or under-treated BD during pregnancy is associated with poor birth outcomes independent of pharmacotherapy exposure, including preterm birth, low birth-weight, intrauterine growth retardation, small for gestational age, fetal distress, and adverse neurodevelopmental outcomes. ${ }^{14}$ Women with untreated BD also have behavioral risk factors such as decreased compliance with prenatal care, poor nutrition, and high-risk behaviors (eg, sexual indiscretions and substance abuse) that jeopardize the fetus. ${ }^{15}$

Impaired capacity to function may result in loss of employment, health care benefits, and social support. The biological and psychosocial risks of a BD episode are the justification for the risk of medication exposure.

Lithium, anticonvulsants (lamotrigine [LTG] and carbamazepine), and second-generation antipsychotics (aripiprazole, asenapine, lurasidone, olanzapine, immediate-release and extended-release quetiapine, risperidone, and ziprasidone) have Food and Drug 
Administration indications for the treatment of BD. Lithium and anticonvulsants are longterm maintenance treatments to prevent manic and depressive episodes. Similarly, secondgeneration antipsychotics are prescribed for maintenance treatment and are also effective treatments for acute mania. Although the anticonvulsant valproic acid is an effective mood stabilizer, other drugs are preferred for women of childbearing age owing to the risk for birth defects and neuro-developmental delays. Carbamazepine is typically continued for patients for whom another option would not be as effective or when the patient presents pregnant and the risk of changing to another drug with unknown efficacy increases the risk for poor outcomes owing to recurrence.

Abrupt discontinuation of psychotropic medications is associated with an increased risk for illness recurrence. Women with BD who discontinue their medications before or during pregnancy have a $71 \%$ risk of recurrence with new episodes occurring most frequently in the first trimester. ${ }^{16}$ Recurrent illness during pregnancy is associated with a $66 \%$ increase in the risk of postpartum episodes. ${ }^{16}$ Women who only have a history of postpartum psychosis are less likely to have a recurrence of BD during pregnancy. ${ }^{17}$ For these women, medication may be tapered and discontinued during pregnancy and resumed immediately postpartum to prevent post-partum psychosis. ${ }^{17}$

There are physiologic changes during pregnancy that result in decreases in the plasma concentrations of many psychotropic medications. Therapeutic drug monitoring is essential for lithium and LTG therapy during the perinatal period. Serial assessment of symptoms across pregnancy is also critical to make dose adjustments. Because depressive episodes are the most common mood recurrences during the perinatal period, assessment with the EPDS or the PHQ-9 is advised at each office visit. Although antidepressants are often used as adjunctive treatment, monotherapy risks mania induction and destabilization for women with bipolar 1 disorder. We summarize treatment recommendations, including symptom and therapeutic dose monitoring, as well as dose adjustments across childbearing for commonly prescribed medications.

\section{Lithium}

Congenital malformations-Fetal exposure to lithium has been associated with an increased risk for cardiac abnormalities. The risk for Ebstein's anomaly with first trimester exposure is $1(0.1 \%)$ to 2 in $1000(0.2 \%)$, but the absolute risk remains low. ${ }^{18}$ Folate supplementation with $5 \mathrm{mg}$ reduces the risk and severity of congenital heart disease by suppressing lithium-induced potentiation of a signaling pathway that inhibits genes important to initiating cardiogenesis. ${ }^{19}$ No other congenital malformations have been associated with lithium exposure.

Infant outcomes-Adverse outcomes of in utero lithium exposure are associated with increased risk for diabetes insipidus, respiratory problems, tachycardia, transient neonatal hypothyroidism, tremor, and neuromuscular complications..$^{20-22}$ The incidences of these outcomes have not been defined and are based on a few case reports. Elevated maternal plasma and umbilical cord lithium concentrations at delivery increase the risk for adverse neonatal outcomes including neuromuscular, central nervous system, and respiratory 
complications. ${ }^{23}$ Children exposed to lithium in utero have not been reported to experience neurodevelopmental delays. ${ }^{24}$

Management during pregnancy and postpartum-Strategies to minimize fetal exposure and maintain efficacy include using the lowest effective dose, prescribing lithium twice daily to avoid high peak serum concentrations, and regular monitoring of lithium serum concentrations. ${ }^{25}$ Lithium has a narrow therapeutic window $(0.6-1.0 \mathrm{mEq} / \mathrm{L})$ and serum concentrations are obtained at 12 hours after dosing for therapeutic dose monitoring. ${ }^{26}$ Lithium is exclusively eliminated by the kidney; as a result, lithium serum concentrations begin decreasing during the first trimester of pregnancy owing to increases in the glomerular filtration rate. ${ }^{27,28}$ The lithiumdose must be increased to maintain effective concentrations. Because the therapeutic lithium concentration varies greatly among women, the effective serum concentration is established before pregnancy. If a therapeutic concentration has not been established, the lithium dose is titrated to a concentration within the therapeutic range. Throughout pregnancy, lithium concentrations are monitored monthly with dose adjustment as appropriate. ${ }^{25}$ For women with first trimester exposure, fetal echocardiography and a level 2 ultrasound examination is recommended at 16 to 18 weeks of gestation to evaluate for anomalies. ${ }^{20,25}$ Holding the lithium dose at the onset of labor or 24 to 48 hours before a planned labor induction or cesarean section prevents adverse outcomes associated with high lithium concentrations at delivery. ${ }^{23}$ After delivery, the lithium dose is immediately adjusted back to the prepregnancy therapeutic dose. If lithium was initiated during pregnancy, lithium levels are checked at the end of the first postpartum week and repeated weekly to achieve a concentration in the therapeutic range and prevent toxicity.

Breastfeeding-Few cases of adverse effects have been reported for newborns exposed to lithium exclusively through breast milk. Published cases have included newborns who had cyanosis, elevated thyroid-stimulating hormone, and lithium toxicity who were also exposed to lithium in utero. ${ }^{29,30}$ In all cases, the adverse effectswere associated with maternal toxicity or compromised health status of the infant (ie, infection, prematurity).

Data from case series estimate that newborns exposed to lithium through breast milk have serum lithium concentrations that are one-fourth of the maternal serum lithium concentration. ${ }^{31}$ LACTMED advises that women who have full-term infants can take lithium and breastfeed with monitoring for infant restlessness or difficulty feeding. ${ }^{32}$ Lithium exposure in infants with an infection, dehydration, or premature birth increases the risk of adverse effects and fluid supplementation and pumping, instead of breastfeeding, is appropriate until the infant is evaluated and lithium toxicity is ruled out. Maternal and infant lithium levels are obtained for an infant with changes in behavior, increased sedation, signs of dehydration, restlessness, and difficulty feeding. ${ }^{33}$ Laboratory monitoring of infant lithium serum concentrations, thyroid-stimulating hormone, blood urea nitrogen, and creatinine are only advised for clinical concerns.

\section{Lamotrigine}

Congenital malformation-LTG exposure has not been associated with an increased risk of congenital malformations. Four epilepsy registries reported the rate of major congenital 
malformation (MCM) to be between $2.0 \%$ and $2.9 \%$ for a combined total of 6498 pregnant women with first trimester exposure to LTG, which is similar to the $3 \%$ to $5 \% \mathrm{MCM}$ rate in the general population. ${ }^{34-37}$ The European Registryof Antiepileptic Drugs and Pregnancy ${ }^{35}$ and the United Kingdom Epilepsy and Pregnancy Register ${ }^{38}$ previously reported a doserelated increase in MCMs for LTG doses greater than $200 \mathrm{mg}$ and of greater than $300 \mathrm{mg} / \mathrm{d}$, respectively but a recent analysis from a larger sample did not replicate a significant difference in the rate of MCMs for low-dose ( $</ 5$ to $200 \mathrm{mg}$ ) versus high-dose ( $>400 \mathrm{mg}$ ) LTG. ${ }^{37}$ A 6-fold increased risk for cleft palate was reported from the North American Pregnancy AED Registry in 2008, ${ }^{39}$ but this finding has not been reproducible. A recent (2016) population-based case-control study did not observe an increase in orofacial cleft or cleft palate compared with babies not exposed to LTG. ${ }^{40}$ Given the favorable reproductive profile of LTG, it is a preferred option among patients and physicians.

Infant outcomes-Pregnancy complications such as preterm birth, miscarriages, and stillbirths have not been associated with in utero LTG exposure. ${ }^{41}$ Studies suggest that IQ scores are average or potentially above average in children with in utero exposure to LTG and there are no significant adverse effects. ${ }^{42,43}$

Management during pregnancy and postpartum-During pregnancy, LTG clearance increases by as much as $330 \% .{ }^{44}$ The accelerated metabolism is attributed to the increasing concentrations of estradiol across pregnancy and the associated upregulation of the primary metabolic enzyme for LTG, uridine diphosphate-glucuronosyl transferase (UGT1A4). Decreasing LTG concentrations across pregnancy owing to increased clearance requires dose adjustments to maintain therapeutic LTG concentrations. In nonpregnant patients, the recommended maintenance dose of LTG for BD is $200 \mathrm{mg}$, but effective doses range from 100 to $500 \mathrm{mg} .{ }^{45,46}$ During pregnancy, patients may require double to triple their prepregnancy dose. Because a therapeutic range for LTG has not been determined for the treatment of BD and is variable among individuals, a woman's effective concentration is determined by checking her trough LTG serum concentration (ie, the lowest serum concentration of a drug, which is right before the next dose is scheduled) before pregnancy. 47

LTG concentrations begin decreasing as early as 10 weeks of gestation and plateau in the third trimester. Monthly monitoring of trough concentrations is necessary to make timely dose adjustments. ${ }^{47}$ If more convenient for the patient, blood draws may be obtained at a consistent time of day, instead of at trough, for comparability between timepoints. Patients are also advised to take LTG twice a day and at the same time each day to maintain stable concentrations.

If the LTG dose is increased during pregnancy, an immediate taper to the prepreg-nancy dose within 2 weeks postpartum is critical owing to the rapid decline in LTG clearance postpartum. Based on an adaptation of neurology clinical guidelines ${ }^{48,49}$ we recommend an immediate postbirth decrease in dose by $25 \%$ to prevent toxicity symptoms of ataxia, dizziness and double or blurred vision as well as prevent the transfer of high concentrations of LTG through breastfeeding. The dose must be further decreased every 3 to 4 days until the prepregnancy dose is reached. ${ }^{49}$ 
Breastfeeding-Maternal-to-infant transfer of LTG in breast milk has been reported in 40 cases and plasma concentrations in infants are $6 \%$ to $50 \%$ of the maternal plasma concentration. ${ }^{47}$ Although infants do not have the full capacity to metabolize LTG by glucuronidation until 6 months of age, ${ }^{50}$ there are no known contraindications to breastfeeding in full-term, healthy neonates. ${ }^{51}$ There have been no reports of rashes associated with breast milk exposure to LTG. Only 1 case reported by Nordmo and colleagues $^{52}$ (2009) of an adverse effect associated with breast milk exposure to LTG has been published. The mother had toxic levels of LTG and exposed her infant through breast milk. This report underscores the importance of dose adjustments back to the prepregnancy baseline dose postpartum. If a woman taking LTG breast-feeds and her infant has difficulty feeding or is lethargic, obtaining an evaluation of the infant by a pediatrician as well as a maternal and infant LTG serum concentration rules out toxicity. Neurodevelopment at 6 years of age for children exposed to LTG through breast milk after exposure in utero is not significantly different from children without breast milk exposure to LTG after exposure in utero. Infants who were breastfed had higher IQ scores on average. ${ }^{53}$

\section{Carbamazepine}

Congenital malformation-Carbamazepine therapy during pregnancy is associated with an increase in congenital malformations. In a metaanalysis of 21 prospective studies and 1255 exposures to carbamazepine in utero, $6.7 \%$ of infants had a major defect compared with $2.34 \%$ of controls. ${ }^{54}$ In a majority of the studies, women had first trimester exposure. The most common malformations associated with carbamazepine monotherapy were neural tube, cardiac, and urinary tract defects. First trimester exposure, specifically, was linked to fingernail hypoplasia, developmental delays, and craniofacial malformations. Treatments combining carbamazepine with other anticonvulsants increased the risk for malformation and taking 2 additional antiepileptic drugs were linked to cardiac, cleft palate, and urinary tract abnormalities. ${ }^{55}$ Oxcarbazepine is an anticonvulsant with off-label use commonly used for $\mathrm{BD}$ and is thought to have less teratogenic effects than carbamazepine, although more investigation is needed.

Infant outcomes-Carbamazepine exposure in utero is linked to reduced fetal head growth. ${ }^{54,56,57}$ Although carbamazepine is associated with restricted intrauterine growth, birth weight, and body length in some studies, ${ }^{58}$ other investigations have not found these variables to differ significantly from controls. ${ }^{56}$ Two reports of toxicity have been published including a case of transient hepatic toxicity and hyperbilirubinemia. ${ }^{59}$

Exposure to carbamazepine in utero has not been associated with an effect on IQ in prospective, retrospective, or registry-based studies ${ }^{60} \mathrm{~A}$ decrease in verbal ability compared with controls was found in a recent prospective study (2015) of children born to women with epilepsy and blindly assessed at 6 years of age after exposure to carbamazepine during gestation. ${ }^{43}$ This finding contradicted a previous report that verbal IQ was not significantly different from controls in a similarly designed investigation. ${ }^{61}$

Management during pregnancy and postpartum—During pregnancy, unbound and free carbamazepine serum levels do not significantly change and therapeutic drug 
monitoring is not clinically useful. ${ }^{62}$ Because carbamazepine therapy is associated with vitamin $\mathrm{K}$ deficiency, experts recommend women take $20 \mathrm{mg} / \mathrm{d}$ of oral vitamin $\mathrm{K}$ during the last month of pregnancy. ${ }^{25}$ Vitamin $\mathrm{K}$ should also be administered to the newborn, $1 \mathrm{mg}$ intramuscularly.

Breastfeeding-For women taking carbamazepine and breastfeeding, 32\% to $80 \%$ of the maternal serm carbamazepine is found in the breast milk. Interindividual variability is wide because the active metabolite is poorly excreted in the breast milk. Breastfeeding is supported for women taking carbamazepine, but any concerns for adequate growth or lethargy are evaluated by the pediatrician, including checking of maternal and infant serum concentrations. ${ }^{63,64}$

\section{Second-generation antipsychotics}

Second-generation antipsychotics have Food and Drug Administration indications for the acute management of psychotic illnesses, acute mania, and maintenance treatment for BD. Additionally, they are combined with mood stabilizers for patients who have achieved a partial response despite dose optimization. With the increasing number of second-generation antipsychotics on the market, fewer first-generation anti-psychotic medications (eg, haloperidol, perphenazine, fluphenazine) are prescribed. This trend is due to the occurrence of fewer extrapyramidal side effects and increased patient tolerability compared with firstgeneration antipsychotics. Although first-generation antipsychotics are effective and have more published data during pregnancy, we focus on second-generation antipsychotics because they are more commonly prescribed and have more than doubled in use during pregnancy in the United States. ${ }^{65}$

Congenital malformation-Second-generation antipsychotics are not associated with an increased risk of MCMs when compared with healthy controls, with the exception of risperidone. An increased risk for cardiac malformation with a relative risk of 1.26 after adjustment for potential confounding variables has been reported for risperi-done but more investigation is needed. ${ }^{66}$ The national pregnancy registry data for second-generation antipsychotics from the Massachusetts General Hospital has had 303 women with secondgeneration antipsychotic exposure complete the study and the risk of major malformation was not found to be beyond that of the general population. ${ }^{67} \mathrm{~A}$ recent investigation of 9258 women enrolled in Medicaid with a live-born infant and at least 1 filled prescription for a second-generation antipsychotic during pregnancy did not find an increased risk for congenital malformations. ${ }^{66}$ Among women not exposed to a second-generation antipsychotic during pregnancy, 32.7 per 100 births had a congenital malformation compared with 44.5 per 1000 births among women who took a second-generation antipsychotic. The rate of malformation is consistent with the $3 \%$ to $5 \%$ rate in the general population. More infant developmental outcome data related to general second-generation anti-psychotic use and individual second-generation antipsychotic medications are needed.

Infant outcomes-Some investigators report an increased risk of low birth weight and small for gestational age in babies exposed to second-generation antipsychotics in utero. ${ }^{68,69}$ In many studies, the effect of illness exposure on birth outcomes has not been determined. 
Lin and colleagues ${ }^{68}$ (2010) notd that the risk for low birth weight, preterm birth, small for gestational age, and large for gestational age was similar in women with schizophrenia who were treated with a second-generation antipsychotic compared with untreated women with schizophrenia. An increased risk of obesity owing to second-generation antipsychoticrelated weight gain and gestational diabetes has been reported as an independent risk factor of second-generation antipsychotic treatment and increases the risk of poor outcomes such as macrosomia, impaired growth, and preterm birth. ${ }^{69-71}$ The weight-neutral properties of aripiprazole and lurasidone make them preferred second-generation anti-psychotics during the perinatal period. Alternatively, olanzapine and quetiapine are associated with excessive weight gain, which may increase the risk for poor birth outcomes. Consistent with the recommendations from the American College of Obstetricians and Gynecologists for obesity management, monitoring body mass index beginning at the first prenatal visit and reinforcing weight management techniques of dietary control, exercise, and behavior modification are necessary to prevent excessive weight gain. High-risk obstetric care is recommended. Patient referrals to the National Registry at Massachusetts General Hospital are encouraged to obtain more data on pregnancy outcomes, which will help to inform reproductive risk. ${ }^{72}$

In a small study of infants, in utero second-generation antipsychotic exposure was associated with a delay in cognitive, motor, and social/emotional development at 2 months. ${ }^{73}$ At 6 and 12 months of age, no delay was present. In a prospective study of controls versus infants exposed to a second-generation antipsychotic, no significant differences were found at 2 months of age for cognitive, motor, and social/emotional functioning or for language. ${ }^{74}$

Breastfeeding-Olanzapine, risperidone, and quetiapine have the most available data to inform exposure risk during breastfeeding. These agents have milk/plasma ratios (the estimate of concentration in maternal serum to milk) that are less than 1 , and relative infant dose ratios that are less than $10 \%$, reflecting acceptable infant exposure. ${ }^{75}$ Information on maternal serum-to-milk transfer is limited for other second-generation antipsychotics such as lurasidone and aripiprazole. Although prevalence has not been established, adverse events are infrequent, mild, and include somnolence, irritability, tremor, and insomnia as well as speech, motor, and mental delays.

Dose management during pregnancy and postpartum-Doses of secondgeneration antipsychotics may need to be increased owing to the increased metabolism by the cytochrome P450 system including CYP2D6 and CYP3A4 enzymes. ${ }^{76}$ During pregnancy, enzyme activity of CYP2D6 increases beginning early in the second trimester and increases further as the pregnancy progresses. ${ }^{77}$ Risperidone is predominantly metabolized by CYP2D6 and may decrease in concentration across pregnancy, resulting in a need for a dose increase. Lurasidone, aripiprazole, and quetiapine concentrations may also decrease across gestation because they are substrates of CYP3A4, which is also induced in pregnancy. ${ }^{77,78}$ Becausea therapeutic concentration is not established for second-generation antipsychotics and laboratory tests to check serum concentrations are not standard, symptom assessment at each office visit informs the need for a dose adjustment. Worsening symptoms for patients who were previously taking a second-generation antipsychotic and without 
symptoms suggest a decline in the preconception serum concentration and requires a dose increase. Postpartum, doses must be decreased to the original preconception dose to prevent adverse side effects and toxicity.

\section{NONPHARMACOLOGIC TREATMENT}

\section{Bright Light Therapy}

Bright light therapy is an evidenced-based treatment for seasonal depression that consists of exposure to broad spectrum light. Although many women with BD are unable to use alternatives to pharmacotherapy as their only treatment, bright light therapy is an effective augmentation strategy for the treatment of residual bipolar depression symptoms in patients taking an antimanic agent. It has not been associated with any reproductive risks and is an acceptable treatment among pregnant women. ${ }^{79}$ The mechanism for symptom reduction is unclear and is attributed to the stabilization of the circadian rhythm and to a modulating effect on neurotransmitters such as 5HT that impact depression. Like all antidepressant treatments, light therapy can precipitate mania in women. It is contraindicated for women in manic, hypomanic, or mixed (ie, both depressive and manic symptoms) mood states. Patients who report excessive irritability are often in a mixed episode and, in these instances, light therapy is avoided. Side effects of bright light therapy include headache, nausea, and jitteriness.

The evidence-based apparatus for light therapy is a fluorescent box with a broad spectrum, ultraviolet-blocked light that provides 10,000 lux and is available commercially. In a recent double-blinded randomized controlled trial, Sit and colleagues ${ }^{80}$ found that adjunctive midday bright light therapy with 7000 lux remitted depressive symptoms in 68\% of depressed nonpregnant adults with bipolar 1 and bipolar 2 disorder compared with $22 \%$ in the control group. None of the patients in the treatment group experienced an onset of manic symptoms. Per Sit and colleagues' findings, therapy is recommended as follows:

1. With concurrent use of an antimanic agent,

2. Midday (between 12:00 PM and 2:30 PM),

3. Starting with 15 minutes a day for week one and increasing by 15 minutes a day each week until 60 minutes is reached at week 4 , and

4. For a duration of 6 weeks.

Women with bipolar depression sit 12 to 14 inches away from the box, which is situated at or above eye-level. Additional information can be found on the nonprofit Center for Environmental Therapeutics (www.CET.org).

\section{TREATMENT RESISTANCE}

\section{Electroconvulsive Therapy}

Electroconvulsive therapy is a longstanding, safe, and effective procedure of passing electric currents through the brain to reduce severe mental health symptoms. Electro-convulsive therapy is completed under general anesthesia and is an effective option for perinatal 
patients who have a severe mood episode and have not responded to medication, lack ability for self-care (ie, unable to get out of bed, eat, or drink), are suicidal, or are psychotic. ${ }^{81}$ Prolonged debilitations may jeopardize the fetus or newborn owing to poor self-care and functional incapacity. We recommend that patients who have not responded to medication after 3 medication trials or are an imminent risk to themselves have a consultation with a psychiatrist and be considered for electro-convulsive therapy.

\section{SUMMARY}

The perinatal period is a vulnerable time for the onset or reemergence of BD symptoms, especially those of bipolar depression. Screening with the MDQ in addition to the EPDS or PHQ-9 depression screen is a critical step in differentiating unipolar depression from bipolar depression to establish an optimal treatment plan. Pharmacotherapy is the most effective treatment for most women with $\mathrm{BD}$, and the risks and benefits of exposure to medication versus untreated illness during pregnancy must be considered for each woman. Women with $\mathrm{BD}$ who continue medication in pregnancy and postpartum reduce their risk for episode recurrence as well as the onset of postpartum depression and postpartum psychosis. If available and indicated, medication combined with bright light therapy optimizes treatment for women with bipolar depression. When psychiatric services are limited, use of professional and patient mental health resources as shown in Box 1 are advised.

\section{REFERENCES}

1. Merikangas KR, Akiskal HS, Angst J, et al. Lifetime and 12-month prevalence of bipolar spectrum disorder in the National Comorbidity Survey replication. Arch Gen Psychiatry 2007;64(5):543-52. [PubMed: 17485606]

2. Leibenluft E Issues in the treatment of women with bipolar illness. J Clin Psychiatry 1997;58(Suppl 15):5-11.

3. Sit DK, Wisner KL. Identification of postpartum depression. Clin Obstet Gynecol 2009;52(3):45668. [PubMed: 19661761]

4. Terp IM, Mortensen PB. Post-partum psychoses. Clinical diagnoses and relative risk of admission after parturition. Br J Psychiatry 1998;172(6):521-6. [PubMed: 9828994]

5. Cantwell R, Clutton-Brock T, Cooper G, et al. Saving mothers' lives: reviewing maternal deaths to make motherhood safer: 2006-2008. The eighth report of the confidential enquiries into maternal deaths in the United Kingdom. BJOG 2011;118(Suppl 1):1-203.

6. American Psychiatric Association. Diagnostic and statistical manual of mental disorders. 5th edition Washington, DC: American Psychiatric Publishing; 2013.

7. O'Connor E, Rossom RC, Henninger M, et al. Primary care screening for and treatment of depression in pregnant and postpartum women: evidence report and systematic review for the US Preventive Services Task Force. JAMA 2016; 315(4):388-406. [PubMed: 26813212]

8. Wisner KL, Sit DK, McShea MC, et al. Onset timing, thoughts of self-harm, and diagnoses in postpartum women with screen-positive depression findings. JAMA Psychiatry 2013;70(5):490-8. [PubMed: 23487258]

9. Cox JL, Holden JM, Sagovsky R. Detection of postnatal depression. Development of the 10-item Edinburgh Postnatal Depression Scale. Br J Psychiatry 1987;150:782-6. [PubMed: 3651732]

10. Kroenke K, Spitzer RL, Williams JB. The PHQ-9: validity of a brief depression severity measure. J Gen Intern Med 2001;16(9):606-13. [PubMed: 11556941]

11. Hirschfeld RM, Williams JB, Spitzer RL, et al. Development and validation of a screening instrument for bipolar spectrum disorder: the Mood Disorder Questionnaire. Am J Psychiatry 2000;157(11):1873-5. [PubMed: 11058490] 
12. Merrill L, Mittal L, Nicoloro J, et al. Screening for bipolar disorder during pregnancy. Arch Womens Ment Health 2015;18(4):579-83. [PubMed: 25968603]

13. Clark CT, Sit DK, Driscoll K, et al. Does screening with the MDQ and EPDS improve identification of bipolar disorder in an obstetrical sample? Depress Anxiety 2015;32(7):518-26. [PubMed: 26059839]

14. Jablensky AV, Morgan V, Zubrick SR, et al. Pregnancy, delivery, and neonatal complications in a population cohort of women with schizophrenia and major affective disorders. Am J Psychiatry 2005;162(1):79-91. [PubMed: 15625205]

15. Leight KL, Fitelson EM, Weston CA, et al. Childbirth and mental disorders. Int Rev Psychiatry 2010;22(5):453-71. [PubMed: 21047159]

16. Viguera AC, Whitfield T, Baldessarini RJ, et al. Risk of recurrence in women with bipolar disorder during pregnancy: prospective study of mood stabilizer discontinuation. Am J Psychiatry 2007;164(12):1817-24 [quiz: 1923]. [PubMed: 18056236]

17. Bergink V, Bouvy PF, Vervoort JS, et al. Prevention of postpartum psychosis and mania in women at high risk. Am J Psychiatry 2012;169(6):609-15. [PubMed: 22407083]

18. Cohen LS, Friedman JM, Jefferson JW, et al. A reevaluation of risk of in utero exposure to lithium. JAMA 1994;271(2):146-50. [PubMed: 8031346]

19. Huhta JC, Linask K. When should we prescribe high-dose folic acid to prevent congenital heart defects? Curr Opin Cardiol 2015;30(1):125-31. [PubMed: 25389654]

20. Diav-Citrin O, Shechtman S, Tahover E, et al. Pregnancy outcome following in utero exposure to lithium: a prospective, comparative, observational study. Am J Psychiatry 2014;171(7):785-94. [PubMed: 24781368]

21. Blake LD, Lucas DN, Aziz K, et al. Lithium toxicity and the parturient: case report and literature review. Int J Obstet Anesth 2008;17(2):164-9. [PubMed: 18308554]

22. Kozma $\mathrm{C}$ Neonatal toxicity and transient neurodevelopmental deficits following prenatal exposure to lithium: another clinical report and a review of the literature. Am J Med Genet A 2005;132A(4): 441-4. [PubMed: 15633173]

23. Newport DJ, Viguera AC, Beach AJ, et al. Lithium placental passage and obstetrical outcome: implications for clinical management during late pregnancy. Am J Psychiatry 2005;162(11):216270. [PubMed: 16263858]

24. van der Lugt NM, van de Maat JS, van Kamp IL, et al. Fetal, neonatal and developmental outcomes of lithium-exposed pregnancies. Early Hum Dev 2012;88(6): 375-8. [PubMed: 22000820]

25. Yonkers KA, Wisner KL, Stowe Z, et al. Management of bipolar disorder during pregnancy and the postpartum period. Am J Psychiatry 2004;161(4):608-20. [PubMed: 15056503]

26. Deligiannidis KM, Byatt N, Freeman MP. Pharmacotherapy for mood disorders in pregnancy: a review of pharmacokinetic changes and clinical recommendations for therapeutic drug monitoring. J Clin Psychopharmacol 2014;34(2):244-55. [PubMed: 24525634]

27. Schou M, Amdisen A, Steenstrup OR. Lithium and pregnancy. II. Hazards to women given lithium during pregnancy and delivery. Br Med J 1973;2(5859): 137-8. [PubMed: 4699591]

28. Wesseloo R, Wierdsma AI, van Kamp IL, et al. Lithium dosing strategies during pregnancy and the postpartum period. Br J Psychiatry 2017;211(1):31-6. [PubMed: 28673946]

29. Tunnessen WW Jr, Hertz CG. Toxic effects of lithium in newborn infants: a commentary. J Pediatr 1972;81(4):804-7. [PubMed: 5074360]

30. Skausig OB, Schou M. Breast feeding during lithium therapy. Ugeskr laeger 1977;139(7):400-1 [in Danish]. [PubMed: 841726]

31. Viguera AC, Newport DJ, Ritchie J, et al. Lithium in breast milk and nursing infants: clinical implications. Am J Psychiatry 2007;164(2):342-5. [PubMed: 17267800]

32. LactMed. Lithium. Available at: http://toxnet.nlm.nih.gov/cgi-bin/sis/search2/r? dbs1lactmed:@term1@DOCNO1293. Accessed June 18, 2018.

33. Bogen DL, Sit D, Genovese A, et al. Three cases of lithium exposure and exclusive breastfeeding. Arch Womens Ment Health 2012;15(1):69-72. [PubMed: 22277970]

34. Hernandez-Diaz S, Smith CR, Shen A, et al. Comparative safety of antiepileptic drugs during pregnancy. Neurology 2012;78(21):1692-9. [PubMed: 22551726] 
35. Tomson T, Battino D, Bonizzoni E, et al. Dose-dependent risk of malformations with antiepileptic drugs: an analysis of data from the EURAP epilepsy and pregnancy registry. Lancet Neurol 2011;10(7):609-17. [PubMed: 21652013]

36. Cunnington MC, Weil JG, Messenheimer JA, et al. Final results from 18 years of the International Lamotrigine Pregnancy Registry. Neurology 2011;76(21): 1817-23. [PubMed: 21606453]

37. Campbell E, Kennedy F, Russell A, et al. Malformation risks of antiepileptic drug monotherapies in pregnancy: updated results from the UK and Ireland Epilepsy and Pregnancy Registers. J Neurol Neurosurg Psychiatry 2014;85(9):1029-34. [PubMed: 24444855]

38. Morrow J, Russell A, Guthrie E, et al. Malformation risks of antiepileptic drugs in pregnancy: a prospective study from the UK Epilepsy and Pregnancy Register. J Neurol Neurosurg Psychiatry 2006;77:193-8. [PubMed: 16157661]

39. Holmes LB, Baldwin EJ, Smith CR, et al. Increased frequency of isolated cleft palate in infants exposed to lamotrigine during pregnancy. Neurology 2008;70(22 Pt 2):2152-8. [PubMed: 18448870]

40. Dolk H, Wang H, Loane M, et al. Lamotrigine use in pregnancy and risk of orofacial cleft and other congenital anomalies. Neurology 2016;86(18):1716-25. [PubMed: 27053714]

41. Pariente G, Leibson T, Shulman T, et al. Pregnancy outcomes following in utero exposure to lamotrigine: a systematic review and meta-analysis. CNS Drugs 2017;31(6):439-50. [PubMed: 28434134]

42. Rihtman T, Parush S, Ornoy A. Developmental outcomes at preschool age after fetal exposure to valproic acid and lamotrigine: cognitive, motor, sensory and behavioral function. Reprod Toxicol 2013;41:115-25. [PubMed: 23791930]

43. Baker GA, Bromley RL, Briggs M, et al. IQ at 6 years after in utero exposure to antiepileptic drugs: a controlled cohort study. Neurology 2015;84(4):382-90. [PubMed: 25540307]

44. Pennell PB, Newport DJ, Stowe ZN, et al. The impact of pregnancy and childbirth on the metabolism of lamotrigine. Neurology 2004;62(2):292-5. [PubMed: 14745072]

45. GlaxoSmithKline. Lamictal Medication Guide. 2016.

46. Clabrese JR, Suppes T, Bowden CL, et al. A double-blind, placebo-controlled, prophylaxis study of lamotrigine in rapid-cycling bipolar disorder. J Clin Psychiatry 2000;61:841-50. [PubMed: 11105737]

47. Clark CT, Klein AM, Perel JM, et al. Lamotrigine dosing for pregnant patients with bipolar disorder. Am J Psychiatry 2013;170(11):1240-7. [PubMed: 24185239]

48. Sabers A Algorithm for lamotrigine dose adjustment before, during, and after pregnancy. Acta Neurol Scand 2012;126(1):e1-4. [PubMed: 22150770]

49. Pennell P, Peng L, Newport D, et al. Lamotrigine in pregnancy: clearance, therapeutic drug monitoring, and seizure frequency. Neurology 2008;70(22 Part 2): 2130-6. [PubMed: 18046009]

50. Strassburg CP, Strassburg A, Kneip S, et al. Developmental aspects of human hepatic drug glucuronidation in young children and adults. Gut 2002;50(2): 259-65. [PubMed: 11788570]

51. LactMed. Lamotrigine. Available at: http://toxnet.nlm.nih.gov/cgi-bin/sis/search2/r? dbs1lactmed:@term1@DOCNO1399. Accessed June 18, 2018.

52. Nordmo E, Aronsen L, Wasland K, et al. Severe apnea in an infant exposed to lamotrigine in breast milk. Ann Pharmacother 2009;43(11):1893-7. [PubMed: 19826099]

53. Meador KJ, Baker GA, Browning N, et al. Breastfeeding in children of women taking antiepileptic drugs: cognitive outcomes at age 6 years. JAMA Pediatr 2014; 168(8):729-36. [PubMed: 24934501]

54. Diav-Citrin O, Shechtman S, Arnon J, et al. Is carbamazepine teratogenic? A prospective controlled study of 210 pregnancies. Neurology 2001;57(2):321-4. [PubMed: 11468320]

55. Matalon S, Schechtman S, Goldzweig G, et al. The teratogenic effect of carbamazepine: a metaanalysis of 1255 exposures. Reprod Toxicol 2002;16(1):9-17. [PubMed: 11934528]

56. Wide K, Winbladh B, Tomson T, et al. Body dimensions of infants exposed to anti-epileptic drugs in utero: observations spanning 25 years. Epilepsia 2000;41(7): 854-61. [PubMed: 10897157] 
57. Almgren M, Kallen B, Lavebratt C. Population-based study of antiepileptic drug exposure in utero-influence on head circumference in newborns. Seizure 2009; 18(10):672-5. [PubMed: 19828334]

58. Bertollini R, Kallen B, Mastroiacovo P, et al. Anticonvulsant drugs in monotherapy. Effect on the fetus. Eur J Epidemiol 1987;3(2):164-71. [PubMed: 3111880]

59. Frey B, Schubiger G, Musy JP. Transient cholestatic hepatitis in a neonate associated with carbamazepine exposure during pregnancy and breast-feeding. Eur J Pediatr 1990;150(2):136-8. [PubMed: 2279511]

60. Haskey C, Galbally M. Mood stabilizers in pregnancy and child developmental outcomes: a systematic review. Aust N Z J Psychiatry 2017;51(11):1087-97. [PubMed: 28825316]

61. Gaily E, Kantola-Sorsa E, Hiilesmaa V, et al. Normal intelligence in children with prenatal exposure to carbamazepine. Neurology 2004;62(1):28-32. [PubMed: 14718692]

62. Johnson EL, Stowe ZN, Ritchie JC, et al. Carbamazepine clearance and seizure stability during pregnancy. Epilepsy Behav 2014;33:49-53. [PubMed: 24632353]

63. Frey B, Braegger CP, Ghelfi D. Neonatal cholestatic hepatitis from carbamaze-pine exposure during pregnancy and breast feeding. Ann Pharmacother 2002; 36(4):644-7. [PubMed: 11918515]

64. Ward RM, Bates BA, Benitz WE, et al. The transfer of drugs and other chemicals into human milk. Pediatrics 2001;108(3):776-89. [PubMed: 11533352]

65. Toh S, Li Q, Cheetham TC, et al. Prevalence and trends in the use of antipsychotic medications during pregnancy in the U.S., 2001-2007: a population-based study of 585,615 deliveries. Arch Womens Ment Health 2013;16(2):149-57. [PubMed: 23389622]

66. Huybrechts KF, Hernandez-Diaz S, Patorno E, et al. Antipsychotic use in pregnancy and the risk for congenital malformations. JAMA Psychiatry 2016;73(9): 938-46. [PubMed: 27540849]

67. Cohen LS, Viguera AC, McInerney KA, et al. Reproductive safety of second-generation antipsychotics: current data from the massachusetts general hospital national pregnancy registry for atypical antipsychotics. Am J Psychiatry 2016; 173(3):263-70. [PubMed: 26441156]

68. Lin HC, Chen IJ, Chen YH, et al. Maternal schizophrenia and pregnancy outcome: does the use of antipsychotics make a difference? Schizophr Res 2010;116(1):55-60. [PubMed: 19896335]

69. Reis M, Källén B. Maternal use of antipsychotics in early pregnancy and delivery outcome. J Clin Psychopharmacol 2008;28(3):279-88. [PubMed: 18480684]

70. Kessing LV, Thomsen AF, Mogensen UB, et al. Treatment with antipsychotics and the risk of diabetes in clinical practice. Br J Psychiatry 2010;197(4):266-71. [PubMed: 20884948]

71. Hull HR, Dinger MK, Knehans AW, et al. Impact of maternal body mass index on neonate birthweight and body composition. Am J Obstet Gynecol 2008;198(4): 416.e1-6. [PubMed: 18279830]

72. National Pregnant Registry for atypical antipshychotics 2016 Available at: http:// womensmentalhealth.org/clinical-and-research-programs/pregnancyregistry/atypicalantipsychotic/. Accessed June 18, 2018.

73. Peng M, Gao K, Ding Y, et al. Effects of prenatal exposure to atypical antipsychotics on postnatal development and growth of infants: a case-controlled, prospective study. Psychopharmacology 2013;228(4):577-84. [PubMed: 23559219]

74. Shao P, Ou J, Peng M, et al. Effects of clozapine and other atypical antipsychotics on infants development who were exposed to as fetus: a post-hoc analysis. PLoS One 2015;10(4):e0123373. [PubMed: 25909513]

75. Uguz F Second-generation antipsychotics during the lactation period: a comparative systematic review on infant safety. J Clin Psychopharmacol 2016;36(3): 244-52. [PubMed: 27028982]

76. Ingelman-Sundberg M Pharmacogenetics of cytochrome P450 and its applications in drug therapy: the past, present and future. Trends Pharmacol Sci 2004; 25(4):193-200. [PubMed: 15063083]

77. Tracy TS, Venkataramanan R, Glover DD, et al. Temporal changes in drug metabolism (CYP1A2, CYP2D6 and CYP3A activity) during pregnancy. Am J obstetrics Gynecol 2005;192(2):633-9.

78. Hebert MF, Easterling T, Kirby B, et al. Effects of pregnancy on CYP3A and P-glycoprotein activities as measured by disposition of midazolam and digoxin: a University of Washington specialized center of research study. Clin Pharmacol Ther 2008;84(2):248-53. [PubMed: 18288078] 
79. Bais B, Kamperman AM, van der Zwaag MD, et al. Bright light therapy in pregnant women with major depressive disorder: study protocol for a randomized, double-blind, controlled clinical trial. BMC Psychiatry 2016;16(1):381. [PubMed: 27821114]

80. Sit DK, McGowan J, Wiltrout C, et al. Adjunctive bright light therapy for bipolar depression: a randomized double-blind placebo-controlled trial. Am J Psychiatry 2017;175(2):131-9. [PubMed: 28969438]

81. Haxton C, Kelly S, Young D, et al. The efficacy of electroconvulsive therapy in a perinatal population: a comparative pilot study. J ECT 2016;32(2):113-5. [PubMed: 26479488] 


\section{KEY POINTS}

- Women with bipolar disorder are vulnerable to episode recurrence during pregnancy and they have an increased risk for postpartum depression and psychosis.

- Pharmacotherapy is the mainstay of treatment for bipolar disorder and the benefits of medication management during pregnancy and lactation often justify the risks.

- Monthly therapeutic drug monitoring with dose adjustment is recommended for patients taking lithium and lamotrigine during pregnancy.

- Bright light therapy is an effective adjunct to treat bipolar depression. 


\section{Box 1}

\section{Professional and patient mental health resources}

OTIS - Mother To Baby (provides professional and patient consultation about medication exposure during pregnancy and while breastfeeding based on evidenced based information. "Fact Sheets" for patients available on the website)

- $\quad$ http://www.mothertobaby.org/

- $\quad$ Toll-Free: 1 to 866 to 626 to 6847

Postpartum Support

- $\quad$ PSI, Postpartum Support International - www.postpartum.net

Women's Mental Health Center at Massachusetts General Hospital (for pregnancy, postpartum, perimenopause, premenstrual dysphoric disorder information)

- https://womensmentalhealth.org/

National Pregnancy Registry for Atypical Antipsychotics Registry, Massachusetts General Hospital

- www.womensmentalhealth.org/pregnancyregistry

- $\quad$ Toll-Free: 1 to 866 to 961 to 2388

Center for Environmental Therapeutics (bright light therapy)

- www.CET.org 


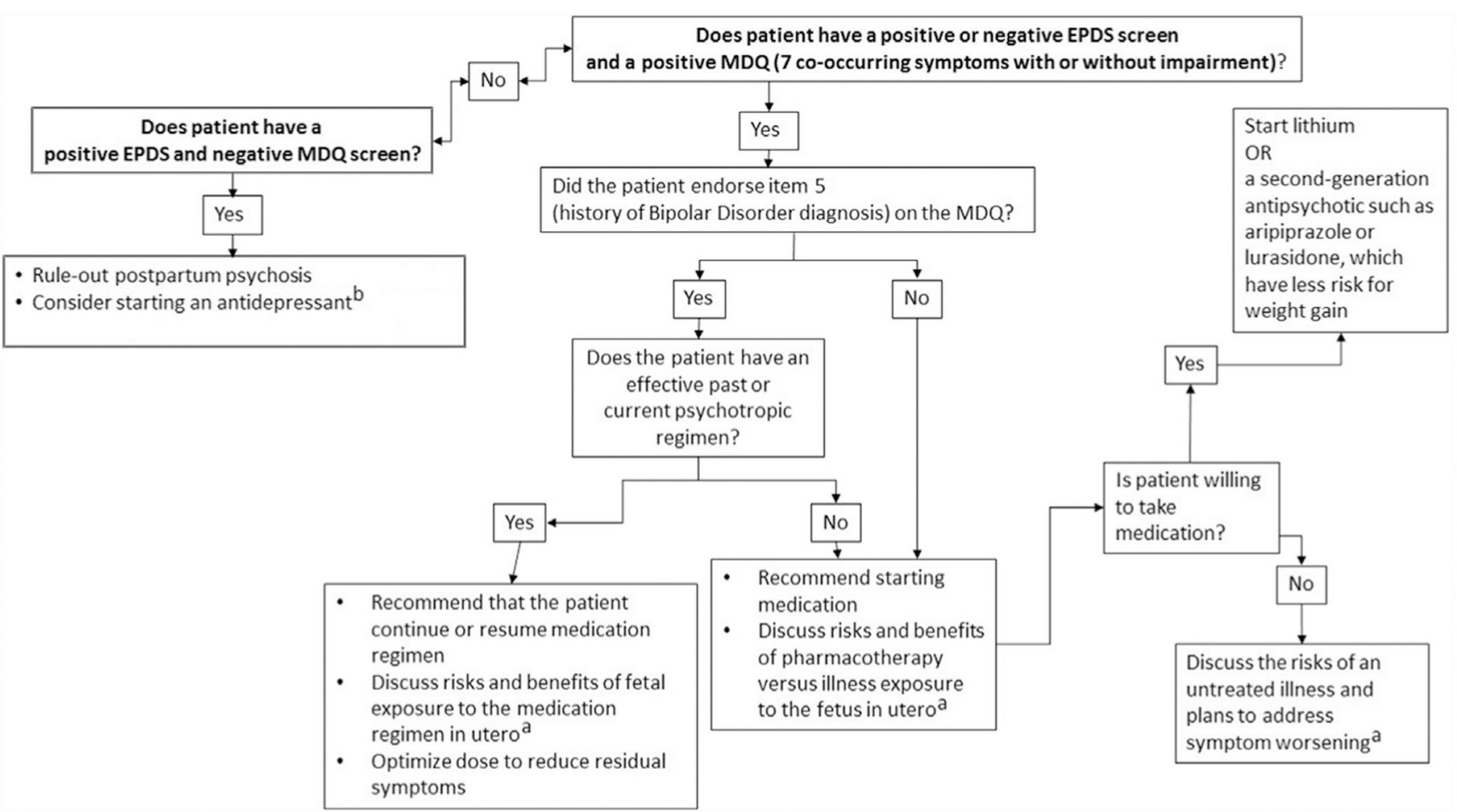

Fig. 1.

Treatment algorithm. ${ }^{\text {a }}$ Additional risk-benefit information and patient Fact Sheetsavailable at Mother to Baby (mothertobaby.org). ${ }^{\mathrm{b}}$ Patients with a family history of bipolardisorder (item 4 of the Mood Disorder Questionnaire [MDQ]) require monitoring for antide pressant induced activation. EPDS, Edinburgh Postnatal Depression Scale. 